\title{
Bioensaios de Atividade Alelopática dos Esteroides ESPINASTEROL, ESPINASTERONA E GLICOPIRANOSIL ESPINASTEROL ${ }^{1}$
}

\author{
Bioassays of Allelopathic Activity of the Steroids Spinasterol, Spinasterone, and Spinasterol \\ Glicopyranosyl
}
RIPARDO FILHO, H.S. ${ }^{2}$, PACHECO, L.C. ${ }^{3}$, SOUZA FILHO, A.P.S. ${ }^{4}$, GUILHON, G.M.S.P. ${ }^{5}$, ARRUDA, M.S.P. ${ }^{5}$ e SANTOS, L.S. ${ }^{5}$

\begin{abstract}
RESUMO - Três esteroides (espinasterol, espinasterona e glicopiranosil espinasterol) foram isolados do caule de Moutabea guianensis, planta de origem amazônica. Suas estruturas foram determinadas a partir das análises de RMN e por comparação com dados espectroscópicos da literatura. Foram avaliadas as atividades alelopáticas das três substâncias em duas plantas invasoras de pastagens comuns da região amazônica: Mimosa pudica (malícia) e Senna obtusifolia (mata-pasto). A substância glicopiranosil espinasterol foi mais ativa no ensaio de desenvolvimento da radícula, inibindo a espécie Senna obtusifolia em $75 \%$; o espinasterol inibiu em $22 \%$ o desenvolvimento do hipocótilo de Senna obtusifolia; e no bioensaio de germinação de sementes as substâncias espinasterol e espinasterona proporcionaram 10\% de inibição em Mimosa pudica. A partir desses resultados, observou-se que a diferença de substituintes observada na posição-3 dos esteroides é capaz de alterar a atividade alelopática. O grupo glicosil na posição-3 elevou a atividade alelopática de forma mais expressiva no bioensaio de desenvolvimento de radícula da espécie malícia, quando comparado aos esteroides hidroxilado e carbonilado. Este é o primeiro estudo químico e alelopático do gênero Moutabea.
\end{abstract}

Palavras-chave: Moutabea guianensis, Polygalaceae, alelopatia, Senna obtusifolia, Mimosa pudica.

\begin{abstract}
Three steroids (spinasterol, spinasterone, and spinasterol glucopyranosyl) were isolated from the stem of the Amazonian plant Moutabea guianensis. Their structures were determined on the basis of NMR analysis and by comparison with spectroscopic data found in the literature. The allelopathic activities of the three substances were evaluated against two common weeds of the Amazon region, Mimosa pudica ('malicia') and Senna obtusifolia ('mata-pasto'). The substance spinasterol glucopyranosyl was the most active in the radicle growth bioassay, inhibiting Senna obtusifolia in 75\%; spinasterol inhibited the development of hypocotyl of Senna obtusifolia in $22 \%$, and spinasterone and spinasterol inhibited $10 \%$ of the seed germination of the species Mimosa pudica. These results showed that the difference of substitutes at the C-3 position of the steroids can influence allelopathic activity: the glicosyl group at the C-3 position enhanced the inhibition of hypocotyl growth of the species Mimosa pudica, compared to the hydroxilated and carbonylated steroid. This is the first chemical and allelopathic study with Moutabea guianensis.
\end{abstract}

Keywords: Moutabea guianensis, Polygalaceae, allelopathy, Senna obtusifolia, Mimosa pudica.

1 Recebido para publicação em 19.11.2011 e aprovado em 21.3.2012.

Parte da dissertação de mestrado do primeiro autor.

2 Doutorando em Química, Programa de Pós-Graduação em Química, Universidade Federal do Pará - UFPA, Rua Augusto Corrêa, S/N, 66075-900 Belém-PA; ${ }^{3}$ Bolsista de Iniciação Científica - CNPq, UFPA; ${ }^{4}$ Eng-ō-Agrōe, Doutor, Embrapa Amazônia Oriental, Trav. Dr. Enéas Pinheiro, S/N, 66095-100 Belém-PA; ${ }^{5}$ Prof. Dr., Programa de Pós-Graduação em Química, UFPA. 


\section{INTRODUÇÃO}

As formas atuais de controle de plantas daninhas, como roçagem (Chiovato et al., 2007; Tipping, 2008), queimadas (Durigan et al., 2007) e utilização de herbicidas sintéticos (Duke et al., 2007), não estão atendendo às atuais necessidades da sociedade, devido às agressões aos recursos naturais (água e solo), à vida silvestre e aos alimentos dos animais e das pessoas, além de favorecer o aumento sistemático no número de espécies de plantas daninhas resistentes aos atuais produtos no mundo (Owen et al., 2007; Powles \& Yu, 2010; Gressel, 2011; Riar et al., 2011). Somada a esses fatores ainda existe a busca dos produtores por herbicidas que possam proporcionar maior produtividade, lucratividade e redução nos gastos com estes produtos. Esses fatores estão forçando os agricultores a buscar novas fontes de herbicidas que atendam às novas exigências.

Aliado a esses aspectos, a percepção de que plantas e microrganismos podem fornecer novas perspectivas de exploração agrícola vem se somar às inúmeras possibilidades de uso da biodiversidade. Especificamente na região amazônica, pela rica e diversificada biodiversidade, as espécies que compõem a floresta tropical podem representar fonte impar para a descoberta de inovadoras e importantes fontes químicas a fo, de para fazer frente ao desafio de se desenvolver a atividade agrícola compativel com as atuais e futuras exigências da sociedade e de mercado (Mourão Junior \& Souza Filho, 2010).

Estudos envolvendo alelopatia, uma subárea da ecologia química, vêm sendo desenvolvidos na tentativa de encontrar possiveis substâncias que possam ser utilizadas pela indústria agroquímica como potentes bioerbicidas.

Entende-se por alelopatia efeitos diretos ou indiretos de uma planta sobre outra, por meio da liberação de substâncias. Esses efeitos podem estimular ou prejudicar o desenvolvimento de espécies sensiveis a essas substâncias - também denominadas de aleloquímicos. Os aleloquímicos podem ser liberados para o meio ambiente de diferentes formas: lixiviação de tecidos vivos e mortos da planta, exsudação radicular, decomposição de tecidos e volatilização (Rice, 1984).

Um dos maiores esforços realizados em estudos envolvendo alelopatia é encontrar maneiras de potencializar os efeitos alelopáticos apresentados por algumas substâncias, a fim de produzir aleloquímicos mais potentes. Uma das formas é inserir modificações estruturais através de semissintese na molécula de interesse e, posteriormente, testar a atividade da molécula modificada. Dentro dessa perspectiva, este trabalho teve como objetivo comparar a atividade alelopática de três esteroides, que possuem o esqueleto estigmasta-7,22-dien, diferenciando-se um do outro apenas por apresentarem diferentes substituintes na posição-3. Assim, foram analisados de que forma esses substituintes são capazes de interferir na atividade alelopática dos esteroides. As três substâncias foram isoladas da espécie amazônica Moutabea guianensis, que pertence à família Polygalaceae - uma família quase cosmopolita que possui cerca de 22 gêneros e 1.000 espécies (Furness \& Sttaford, 1995; Coelho et al., 2008). A espécie $M$. guianensis é conhecida popularmente como cipó-gogó-deguariba, e o seu fruto, como olho-de-abutre ou fruto-de-macaco (Pastore, 2006). Este é o primeiro estudo dessa espécie sob o ponto de vista químico e alelopático. Foram utilizadas como plantas-alvo duas plantas invasoras de pastagens típicas da região amazônica: Mimosa pudica (malícia) e Senna obtusifolia (matapasto).

\section{MATERIAL E MÉTODOS}

\section{Coleta e preparo do material botânico}

O material botânico utilizado (caule de Moutabea guianensis) foi coletado por parataxônomos, e a identificação botânica, feita por botânico do Museu Paraense Emílio Goeldi, onde uma exsicata encontra-se catalogada sob o registro MG 195862.

\section{Isolamento e identificação das substâncias}

O caule seco e moído de Moutabea guianensis foi extraído sucessivamente com hexano, acetato de etila e metanol durante cinco dias, fornecendo os extratos hexânico 
(EHC; 785,00 mg), acetato de etila (EAC; 4,89 g) e metanólico (EMC; 3,12 g). O extrato hexânico foi fracionado em $\mathrm{CC}$ em gel de sílica eluída com misturas de hexano e acetato de etila. Após monitoramento em CCD, as frações de 7-10 eluidas em Hex/AcOEt 10\% foram reunidas e recristalizadas em metanol, de onde foi obtida a substância S1 (espinasterol). O extrato acetato de etila foi fracionado também em CC, tendo como adsorvente gel de sílica e, como eluentes, hexano, acetato de etila e metanol. Após o fracionamento, as frações foram monitoradas por CCD e aquelas com perfis cromatográficos semelhantes foram reunidas. Da fração 3 eluída em Hex/ AcOEt $10 \%$ foi identificada a substância S2 (espinasterona), e da fração 24 eluída em AcOEt/MeOH 50\% foi identificada a substância S3 (glicopiranosil espinasterol). A elucidação estrutural das substâncias isoladas foi realizada pela análise de seus espectros de ressonância magnética nuclear (RMN de ${ }^{1} \mathrm{H}$, RMN de ${ }^{13} \mathrm{C}$, DEPT) e comparação com dados da literatura (Figura 1).

\section{Análise da atividade alelopática}

Bioensaio de germinação de sementes: A germinação foi monitorada em um período de cinco dias, com contagens diárias e eliminação das sementes germinadas. Foram consideradas sementes germinadas aquelas que apresentavam extensão radicular maior ou igual a 2,00 mm (Juntila, 1976; Duram \& Tortosa, 1985). Os bioensaios foram realizados em câmara de germinação em temperatura constante de $25^{\circ} \mathrm{C}$ e fotoperíodo de 12 horas. No bioensaio de germinação de sementes, cada placa de Petri de $9,0 \mathrm{~cm}$ de diâmetro, forrada com um disco de papel-filtro qualitativo, recebeu 15 sementes previamente tratadas com ácido sulfúrico, para a quebra de dormência (Souza Filho et al., 1998).

Bioensaio de desenvolvimento de radicula e hipocótilo: Os bioensaios de desenvolvimento da radícula e do hipocótilo foram realizados em um período de 10 dias. Ao final desse período, mediu-se o comprimento da radícula

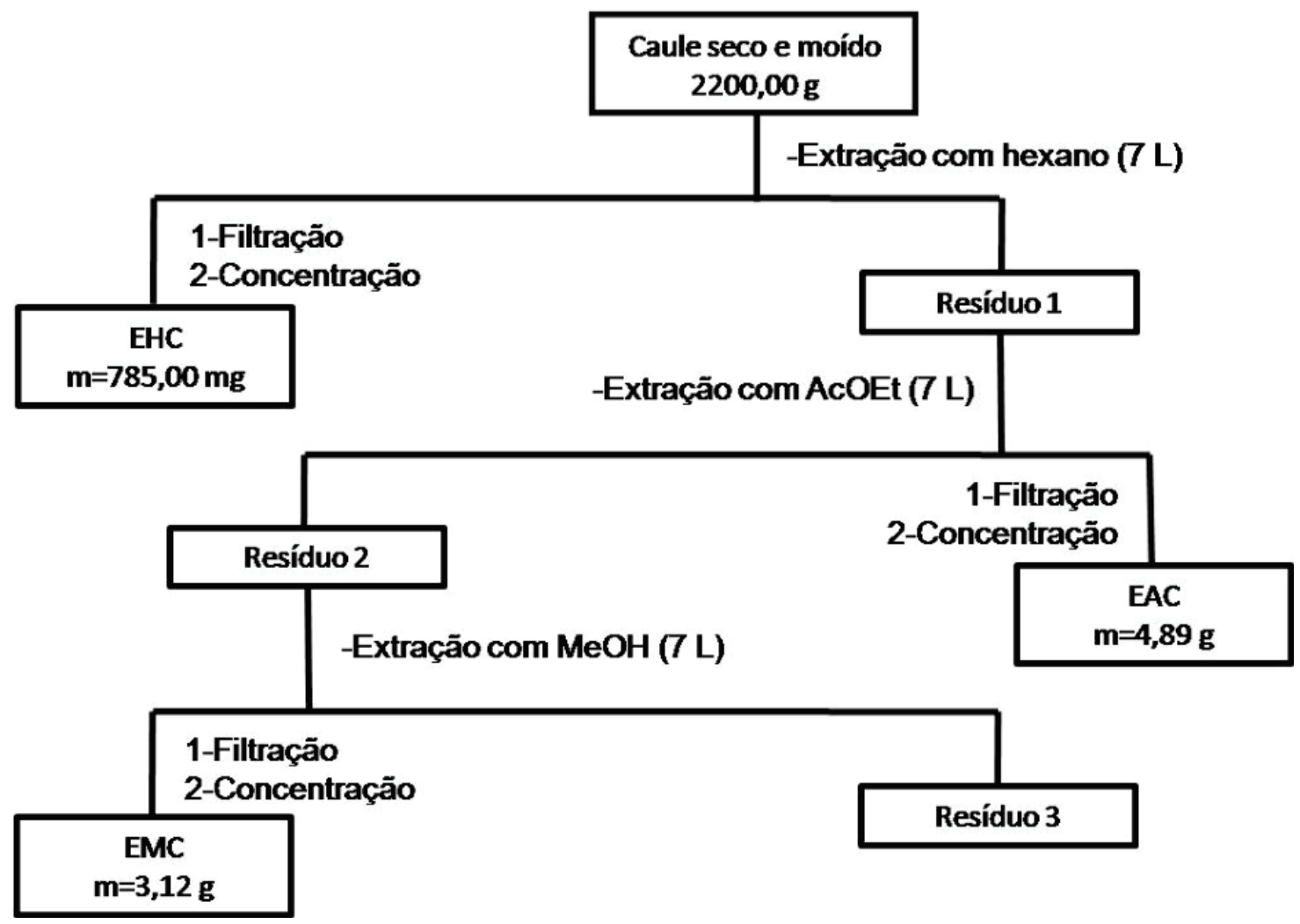

Figura 1 - Fluxograma de obtenção dos extratos do caule de Moutabea guianensis. 
e do hipocótilo. Os bioensaios de desenvolvimento foram realizados em câmara de germinação em temperatura constante de $25{ }^{\circ} \mathrm{C}$ e fotoperiodo de 24 horas. Nos bioensaios de desenvolvimento da radícula e do hipocótilo, cada placa de Petri de $9 \mathrm{~cm}$ de diâmetro, forrada com papel-filtro qualitativo, recebeu duas semente pré-germinadas.

Procedimentos comuns: Em todos os bioensaios, as substâncias S1, S2 e S3 foram testadas separadamente. Cada placa de Petri de $9 \mathrm{~cm}$ de diâmetro recebeu $3 \mathrm{~mL}$ de solução das substâncias-teste, na concentração de $20 \mathrm{mg} \mathrm{L}^{-1}$; as soluções foram adicionadas apenas uma vez, no início dos bioensaios, sendo então adicionada apenas água destilada quando necessário, para manter a concentração inicial.

\section{Delineamento experimental e análise estatística}

O delineamento experimental foi inteiramente casualizado, e as três substâncias foram avaliadas em uma única concentração de $20 \mathrm{mg} \mathrm{L}^{-1}$, com três repetições. Para o experimento, utilizou-se como tratamento testemunha água destilada. Os dados foram analisados pelo teste $\mathrm{F}$, e as médias, comparadas pelo teste de Tukey $(\mathrm{p}>0,05)$. Na análise dos dados utilizou-se o programa SAS (1989).

\section{RESULTADOS E DISCUSSÃO}

\section{Identificação das substâncias}

A substância S1 (Figura 2) foi isolada da fração 12 do extrato hexânico do caule de $M$. guianensis como um sólido branco solúvel em diclorometano e apresentou perfil típico de esteroide no espectro de $\mathrm{RMN}$ de ${ }^{1} \mathrm{H}$, onde é possivel observar, entre outros sinais, um multipleto em $\delta_{\mathrm{H}} 3,60$ característico de hidrogênio oximetínico da posição-3 do esqueleto dos esteroides, dois sinais na região de hidrogênios olefinicos, $\delta_{\mathrm{H}} 5,02(1 \mathrm{H}, d d, J=15,2 \mathrm{~Hz}$, $J=8,5 \mathrm{~Hz}$ ) e uma sobreposição de sinais em $\delta_{\mathrm{H}} 5,15(2 \mathrm{H}, m)$. Nos espectros de RMN de ${ }^{13} \mathrm{C} \mathrm{e}$ DEPT são observados 29 sinais de carbono, dos quais seis são referentes a carbonos metílicos, nove, a metilênicos, dez, a metínicos, e quatro, a quaternários. Desses sinais, podem-se destacar $\delta_{\mathrm{C}} 71,1$ característico de carbono oximetínico em C3 de esteroides e em $\delta_{\mathrm{C}} 117,5, \delta_{\mathrm{C}} 129,4, \delta_{\mathrm{C}} 138,2$ e $\delta_{\mathrm{C}} 139,5$ os sinais de carbonos olefinicos de duas insaturações. Com base nos dados dos espectros de RMN de ${ }^{1} \mathrm{H}$, de ${ }^{13} \mathrm{C}$ e DEPT e comparação com dados da literatura (Zhang et al., 2005), a substância $\mathrm{S} 1$ foi identificada como espinasterol - um esteroide muito encontrado em espécies da família Polygalaceae. Na Tabela 1 é mostrada a comparação dos dados de RMN de ${ }^{13} \mathrm{C}$ obtidos com os dados encontrados na literatura para o espinasterol.

A substância S2 (Figura 2) foi obtida da fração ACC3 do extrato acetato de etila do caule de $M$. guianensis. A substância apresentou-se como um sólido branco solúvel em diclorometano. Em seu espectro de RMN de ${ }^{1} \mathrm{H}$ foram observadas algumas semelhanças com o espectro de RMN de ${ }^{1} \mathrm{H}$ da substância $\mathrm{S} 1$, com a diferença de que neste espectro não foi possivel observar o multipleto referente ao hidrogênio $\mathrm{H}-3$. No espectro de $\mathrm{RMN}$ de ${ }^{13} \mathrm{C}$ foram observados 29 sinais majoritários, dos quais podem-se destacar os sinais de carbonos metínicos $\delta_{\mathrm{C}} 117,1, \delta_{\mathrm{C}} 129,6$ e $\delta_{\mathrm{C}} 138,1$ e o de carbono totalmente substituído $\delta_{\mathrm{C}} 139,6$, que indicam tratar-se de carbonos olefinicos. Pode-se destacar também o sinal em $\delta_{\mathrm{C}} 212,1$, indicativo de carbonila de cetona. A partir das informações acumuladas pelas análises dos espectros de RMN de ${ }^{1} \mathrm{H}$, de ${ }^{13} \mathrm{C}$ e DEPT da substância $\mathrm{S} 1$ analisada anteriormente e comparação com dados da literatura (Souza et al., 2001) (Tabela 1), a substância S2 foi identificada como a espinasterona.

A substância S3 (Figura 2) foi isolada da fração AC24 do extrato acetato de etila do caule de $M$. guianensis como um sólido cristalino solúvel em piridina. O seu espectro de RMN de ${ }^{1} \mathrm{H}$ apresentou-se muito semelhante ao espectro de RMN de ${ }^{1} \mathrm{H}$ da substância $\mathrm{S} 1$, com a diferença de que o multipleto em torno de $\delta_{\mathrm{H}} 3,60$ encontra-se deslocado para $\delta_{\mathrm{H}} 4,00$ e pela presença de sinais de hidrogênio na região de açúcar, sugerindo a presença de uma unidade de açúcar na posição-3. Também podem ser destacados, no espectro de RMN de ${ }^{1} \mathrm{H}$, dois multipletos em $\delta_{\mathrm{H}} 5,02$ e $\delta_{\mathrm{H}} 5,20$ na região de hidrogênios olefinicos. No espectro de RMN de ${ }^{13} \mathrm{C}$ e no espectro de DEPT, foi observada a presença de 35 sinais. Dos 35 sinais, seis são referentes a carbonos metílicos e dez 


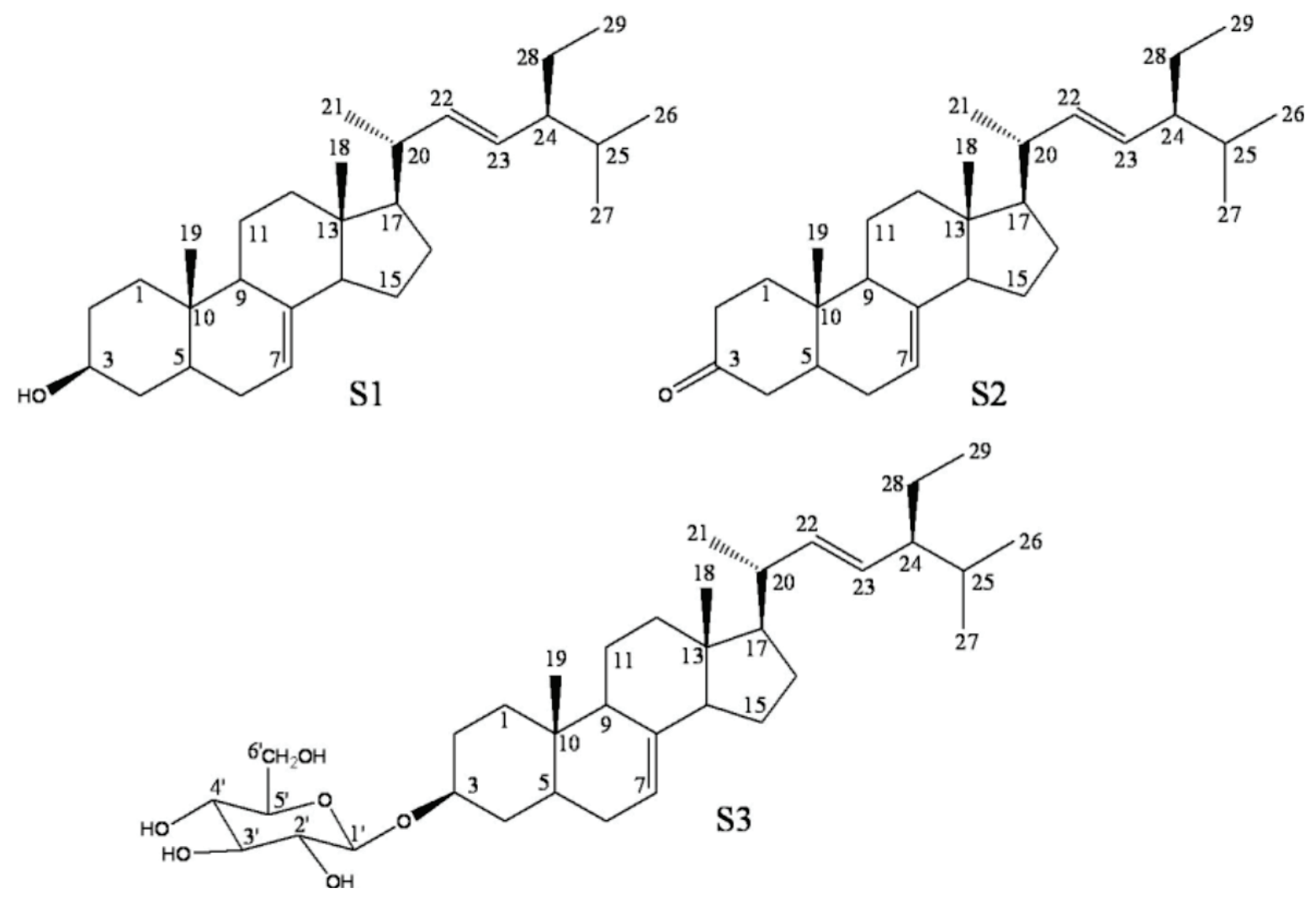

Figura 2 - Estrutura das substâncias isoladas de Moutabea guianensis.

a metilênicos, entre eles $\delta_{\mathrm{c}} 62,9$, característico da posição-6 de unidade de glicose, além de 15 metínicos, dos quais podem-se destacar os sinais de RMN de ${ }^{13} \mathrm{C}$ indicativos de unidade glicosídica $\left(\delta_{\mathrm{C}} 71,8, \delta_{\mathrm{C}} 75,4\right.$, $\delta_{\mathrm{C}} 78,6, \delta_{\mathrm{C}} 78,7$ e $\left.\delta_{\mathrm{C}} 102,3\right)$, o sinal de carbono oximetínico $\left(\delta_{\mathrm{C}} 77,1\right)$ e os sinais presentes na região de carbonos olefinicos $\left(\delta_{\mathrm{C}} 117,9, \delta_{\mathrm{C}} 129,7\right.$ e $\left.\delta_{\mathrm{C}} 138,7\right)$; ainda estão presentes quatro sinais de carbonos quaternários, entre eles aquele localizado na região de carbonos olefínicos: $\delta_{\mathrm{C}} 139,9$. Com as informações obtidas dos espectros de RMN de ${ }^{1} \mathrm{H}$, de ${ }^{13} \mathrm{C}$ e DEPT e comparação com dados da literatura (Zhang et al., 2005), a substância S3 foi identificada como glicopiranosil espinasterol (Tabela 1).

\section{Análise da atividade alelopática das substâncias}

No bioensaio de atividade alelopática sobre a germinação de sementes, foi observado que a espécie Mimosa pudica (malícia) foi a mais sensivel e apresentou maiores inibições diante das substâncias espinasterol (S1) e espinasterona (S2), ambas com 10\% de inibição, enquanto o glicopiranosil espinasterol (S3) inibiu 7\%. Esses resultados são indicativos de que a diferença existente na posição-3 dos esteroides testados (S1-hidroxila, S2-carbonila e S3-glicose) não provocou diferenças significativas nos potenciais de inibição de germinação dessas substâncias na invasora Mimosa pudica (malícia). A espécie Senna obtusifolia (mata-pasto) não se mostrou sensivel ao ensaio de germinação de sementes. Na Figura 3 é apresentado o gráfico com os resultados de inibição de germinação das substâncias $\mathrm{S} 1$, S2 e S3.

A espécie que sofreu maiores inibições no bioensaio de desenvolvimento da radícula (Figura 4) foi Senna obtusifolia (mata-pasto), e a substância que proporcionou maior atividade alelopática para essa invasora foi a glicopiranosil espinasterol (S3), com potencial de inibição de $75 \%$, seguida de espinasterona (S2), com 46\%, e espinasterol (S1), com 37\% 
Tabela 1 - Dados de RMN de ${ }^{13} \mathrm{C}$ de S1 e S2 $\left(75 \mathrm{MHz}, \mathrm{CDCl}_{3}\right)$ e S3 (75 MHz, piridina- $\left.d_{5}\right)$ em comparação com dados da literatura

\begin{tabular}{|c|c|c|c|c|c|c|}
\hline $\mathrm{C}$ & $\delta \mathrm{S} 1$ & $\delta$ S1 Lit. ${ }^{\prime /}$ & $\delta \mathrm{S} 2$ & $\delta$ S2 Lit. ${ }^{2 /}$ & $\delta \mathrm{S} 3$ & $\delta$ S3 Lit. ${ }^{\prime \prime}$ \\
\hline 1 & 37,1 & 37,1 & 38,9 & 38,8 & 37,3 & 37,3 \\
\hline 2 & 31,4 & 31,5 & 38,2 & 38,1 & 30,0 & 30,0 \\
\hline 3 & 71,1 & 71,1 & 212,1 & 212,0 & 77,1 & 77,1 \\
\hline 4 & 37,9 & 38,0 & 44,3 & 44,2 & 34,5 & 34,6 \\
\hline 5 & 40,2 & 40,2 & 43,0 & 42,9 & 40,2 & 40,2 \\
\hline 6 & 29,6 & 29,7 & 30,1 & 30,0 & 30,0 & 30,0 \\
\hline 7 & 117,5 & 117,5 & 117,1 & 117,0 & 117,9 & 117,9 \\
\hline 8 & 139,5 & 139,6 & 139,6 & 139,5 & 139,6 & 139,6 \\
\hline 9 & 49,4 & 49,4 & 49,0 & 48,9 & 49,6 & 49,6 \\
\hline 10 & 34,2 & 34,2 & 34,5 & 34,4 & 34,7 & 34,8 \\
\hline 11 & 21,5 & 21,5 & 21,8 & 21,7 & 21,7 & 21,8 \\
\hline 12 & 39,5 & 39,4 & 39,4 & 39,3 & 39,7 & 39,6 \\
\hline 13 & 43,3 & 43,3 & 43,3 & 43,2 & 43,5 & 43,5 \\
\hline 14 & 55,1 & 55,1 & 55,1 & 55,0 & 55,3 & 55,3 \\
\hline 15 & 23,0 & 23,0 & 23,1 & 22,9 & 23,4 & 23,4 \\
\hline 16 & 28,5 & 28,5 & 28,6 & 28,6 & 28,9 & 29,0 \\
\hline 17 & 55,8 & 55,9 & 56,0 & 56,0 & 56,0 & 56,1 \\
\hline 18 & 12,0 & 12,0 & 12,2 & 12,2 & 12,2 & 12,3 \\
\hline 19 & 13,0 & 13,0 & 12,5 & 12,5 & 13,1 & 13,1 \\
\hline 20 & 40,8 & 40,8 & 40,9 & 40,9 & 41,1 & 41,2 \\
\hline 21 & 21,4 & 21,4 & 21,2 & 21,2 & 21,6 & 21,7 \\
\hline 22 & 138,2 & 138,2 & 138,1 & 138,1 & 138,7 & 138,7 \\
\hline 23 & 129,4 & 129,4 & 129,6 & 129,6 & 129,7 & 129,7 \\
\hline 24 & 51,2 & 51,2 & 51,3 & 51,3 & 51,4 & 51,5 \\
\hline 25 & 31,9 & 31,9 & 32,0 & 32,0 & 32,2 & 32,2 \\
\hline 26 & 19,0 & 19,0 & 21,5 & 21,5 & 19,2 & 19,2 \\
\hline 27 & 21,1 & 21,1 & 19,1 & 19,1 & 21,3 & 21,3 \\
\hline 28 & 25,4 & 25,4 & 25,5 & 25,5 & 25,7 & 25,7 \\
\hline 29 & 12,2 & 12,2 & 12,3 & 12,3 & 12,5 & 12,6 \\
\hline $1^{\prime}$ & & & & & 102,3 & 102,3 \\
\hline $2^{\prime}$ & & & & & 75,4 & 75,4 \\
\hline $3^{\prime}$ & & & & & 78,7 & 78,7 \\
\hline 4 ' & & & & & 71,8 & 71,8 \\
\hline 5 & & & & & 78,5 & 78,6 \\
\hline 6 ' & & & & & 62,9 & 62,9 \\
\hline
\end{tabular}

1/ Zhang et al. (2005). ${ }^{2 /}$ Souza et al. (2001).

de inibição, mostrando que o esteroide substituído na posição-3 pela unidade de açúcar é o mais ativo. A espécie daninha Mimosa pudica (malícia) sofreu maior redução no desenvolvimento da radícula (13\%) diante da substância espinasterol (S1).

No ensaio de desenvolvimento do hipocótilo (Figura 5), a espécie mata-pasto se mostrou mais afetada diante das substâncias espinasterol (S1) e glicopiranosil espinasterol (S3), com potenciais de inibição de 22 e 13\%, respectivamente, mostrando que a presença da hidroxila na posição-3 tem maior influência nessa atividade. A espécie invasora Mimosa pudica (malícia) mostrou-se mais sensivel ao efeito inibitório da substância espinasterol (S1), 9\%.

Trabalhos envolvendo atividade alelopática de esteroides não são frequentes na literatura. Estudos recentes têm demonstrado que substâncias naturais presentes no arroz (Oryza sativa) possuem atividades herbicidas, entre as quais esteroides livres e glicosilados (Chung \& Ahmad, 2010). Os esteroides estigmasterol e $\beta$-sitosterol apresentaram efeitos alelopáticos sobre o crescimento do 


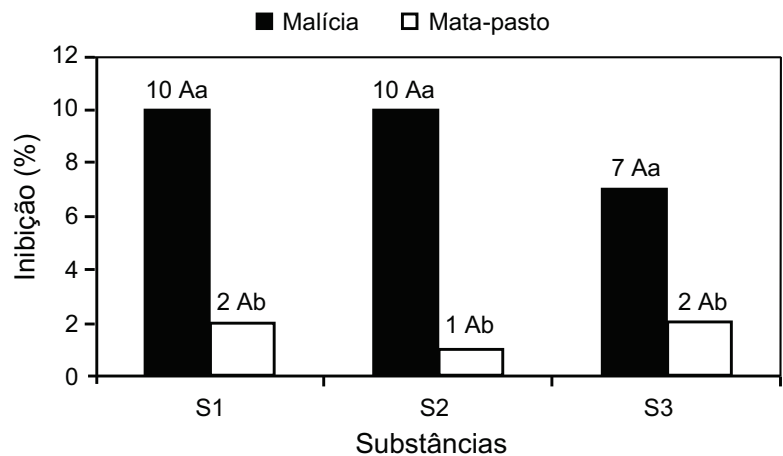

Figura 3 - Efeitos das substâncias $\left(20 \mathrm{mg} \mathrm{L}^{-1}\right)$ testadas sobre a germinação de sementes de Mimosa pudica (malícia) e Senna obtusifolia (mata-pasto). S1: espinasterol; S2: espinasterona; S3: glicopiranosil espinasterol. Dados expressos em percentual de inibição em relação ao tratamento testemunha - água destilada. Médias seguidas de letras iguais, maiúsculas paras as substâncias S1, S2 e S3 e minúsculas para efeito inibitório entre as espécies, não diferem estatisticamente pelo teste de Tukey $(\mathrm{p}>0,05)$.

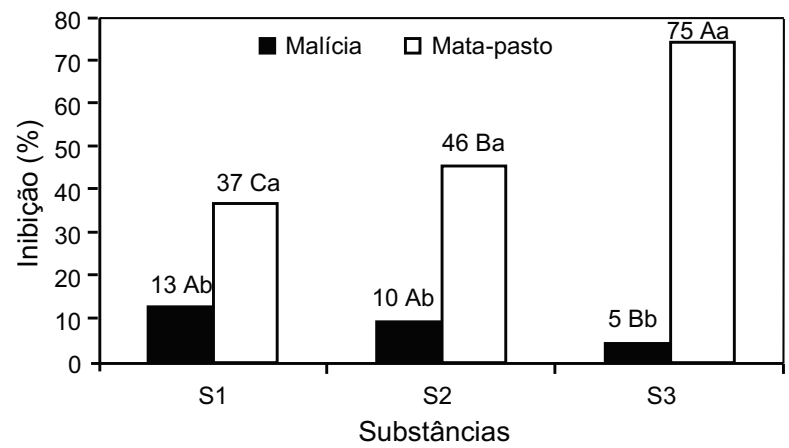

Figura 4 - Efeitos das substâncias $\left(20 \mathrm{mg} \mathrm{L}^{-1}\right)$ sobre o desenvolvimento da radícula de Mimosa pudica (malícia) e Senna obtusifolia (mata-pasto). S1: espinasterol; S2: espinasterona; S3: glicopiranosil espinasterol. Dados expressos em percentual de inibição em relação ao tratamento testemunha-água destilada. Médias seguidas de letras iguais, maiúsculas para as substâncias $\mathrm{S} 1, \mathrm{~S} 2$ e S3 e minúsculas para efeito inibitório entre as espécies, não diferem estatisticamente pelo teste de Tukey $(\mathrm{p}>0,05)$.

feijão-caupi (Vigna unguiculata), mostrando efeito inibitório sobre a taxa de germinação, o comprimento da parte aérea e o peso fresco (Kpoviessi et al., 2006). Macias et al. (1997) demonstraram as atividades alelopáticas de diversos esteroides isolados de Melilotus messanensis sobre a germinação e o crescimento das monocotiledôneas Hordeum vulgare e Allium cepa, bem como das dicotiledôneas
Lactuca sativa (var. Nigra e var. Romana) e Lycopersicon esculentum. Santos et al. (2008a) relataram a atividade alelopática dos esteroides esgosterol e peróxido de ergosterol isolados do fungo endofitico Pestalotiopsis guepinii, cujos resultados se assemelham aos potenciais observados no presente estudo. Em estudo recente (Santos et al., 2008b), foi demonstrado que substâncias que guardam estreitas semelhanças com os esteroides, como os triterpenos, potencializam os efeitos alelopáticos em diferentes $\mathrm{pH}$, e esse é um caminho a ser explorado.

Nos bioensaios de atividade alelopática sobre a germinação de sementes, os esteroides testados não proporcionaram inibições significativas, tendo Mimosa pudica (malícia) e Senna obtusifolia (mata-pasto) como espécies receptoras. Resultados expressivos foram obtidos nos bioensaios de desenvolvimento da radícula, em que a substância glicosilada S3 foi a mais ativa, evidenciando que a substituição na posição-3 dos esteroides pode influenciar no potencial alelopático avaliado. De maneira geral, as atividades alelopáticas foram dosesdependentes e variaram de acordo com a espécie testada e o parâmetro analisado. O fato de triterpenos exibirem atividade alelopática potencializada em diferentes $\mathrm{pH}$ e de a atividade alelopática dos esteroides avaliados no

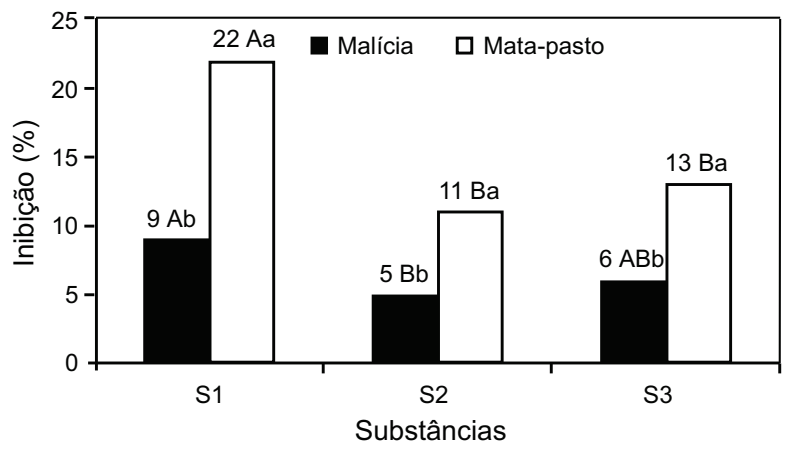

Figura 5 - Efeitos das substâncias $\left(20 \mathrm{mg} \mathrm{L}^{-1}\right)$ sobre o desenvolvimento de hipocótilo de Mimosa pudica (malícia) e Senna obtusifolia (mata-pasto). S1: espinasterol; S2: espinasterona; S3: glicopiranosil espinasterol. Dados expressos em percentual de inibição em relação ao tratamento testemunha-água destilada. Médias seguidas de letras iguais, maiúsculas para as substâncias S1, S2 e S3 e minúsculas para efeito inibitório entre as espécies, não diferem estatisticamente pelo teste de Tukey $(\mathrm{p}>0,05)$. 
presente estudo variar com a substituição na posição-3 abre expectativa para estudos de correlação estrutura química versus atividade biológica, variando os substituintes dessa posição.

\section{AGRADECIMENTOS}

À CAPES e ao CNPq, pelo apoio financeiro.

\section{LITERATURA CITADA}

COELHO, V. P. M.; AGRA, M. F.; BARACHO, G. S. Flora da Paraíba, Brasil: Polygala L. (Polygalaceae). Acta Bot. Bras., v. 22, n. 1, p. 225-239, 2008.

CHIOVATO, M. G. et al. Diferentes densidades de plantas daninhas e métodos de controle nos componentes de produção do milho orgânico. Planta Daninha, v. 25, n. 2, p. 277-283, 2007.

CHUNG, I. M.; AHMAD, A. Herbicidal activities of allelopathic and other compounds from Oryza sativa. Allelopathy J., v. 26, n. 2, p. 139-156, 2010.

DUKE, S. O. et al. Interactions of synthetic herbicides with plant disease and microbial herbicides. In: VURRO, M.; GRESSEL, J. (Eds.). Novel biotechnologies for biocontrol agent enhancement and management. Dordrecht: Springer, 2007. p. 277-296.

DURAM, R. D.; TORTOSA, M. E. The effect of mechanical and chemical scarification on germination of charlock (Sinapis arvensis L.) seeds. Seed Sci. Technol., v. 13, n.1, p. 155-163, 1985.

DURIGAN, G. et al. Threats to the Cerrado remnants of the state of São Paulo, Brazil. Sci. Agric., v. 64, n. 4, p. 355-363, 2007.

FURNESS, S. H.; STAFFORD, P.J. Polygalaceae. Rev. Paleobot. Palynol., v. 88, n. 1, p. 61-82, 1995.

GRESSEL, J. Low pesticide rates may hasten the evolution of resistance by increasing mutation frequencies. Pest Manag. Sci., v. 67, n. 3, p. 253-257, 2011.

JUNTILA, O. Seed and embryo germination in S. vulgaris and $S$. reflexa as affected by temperature during seed development. Physiol. Plant, v. 29, n. 2, p. 264-268, 1976.

KPOVIESSI, D. S. S. et al. Allelopathic effects on cowpea (Vigna unguiculata (L.) Walp) plant and cytotoxic activities of sterols and triterpene isolated from Justicia anselliana (NEES) T. Anders. Electron. J. Nat. Subs., v. 1, n. 1, p. 12-19, 2006.

Planta Daninha, Viçosa-MG, v. 30, n. 4, p. 705-712, 2012
MACIAS, F. A.; SIMONET, A. M.; GALINDO, J. C. G. Bioactive steroids and triterpenes from Melilotus messanensis and their allelopathic potential. J. Chem. Ecol., v. 23, n. 7, p. 1781-1803, 1997.

MOURÃO JUNIOR, M.; SOUZA FILHO, A. P. S. Diferenças da atividade alelopática em espécies da família Leguminosae. Planta Daninha, v. 28, p. 939-951, 2010. Edição Especial.

OWEN, M. J. Widespread occurrence of multiple herbicide resistance in Western Australian annual ryegrass (Lolium rigidum) populations. Austr. J. Agric. Res., v. 58, n. 7, p. 711-718, 2007.

PASTORE, J. F. B. Polygalaceae Hoffmannsegg e Link no Distrito Federal, Brasil. 2006. 216 f. Dissertação (Mestrado em Botânica) - Instituto de Ciências Biológicas, Universidade de Brasília, Brasília, 2006.

POWLES, S. B.; YU, Q. Evolution in action: plants resistant to herbicides. Ann. Rev. Plant Biol., v. 61, n. 1, p. 317-347, 2010 .

RIAR, D. S. et al. Glyphosate resistance in a johnson grass (Sorghum halepense) biotype from Arkansas. Weed Sci., v. 59, n. 3, p. 299-304, 2011.

RICE, E. L. Allelopathy. New York: Academic Press, 1984. $422 \mathrm{p}$.

SANTOS, L. S. et al. Potencial herbicida da biomassa e de substâncias químicas produzidas pelo fungo endofítico Pestalotiopsis guepinii. Planta Daninha, v. 26, n. 3, p. 539-548, 2008a.

SANTOS, L. S. et al. Atividade alelopática de substâncias químicas isoladas do capim-marandu e suas variações em função do pH. Planta Daninha, v. 26, n. 3, p. 531-538, 2008b.

\section{STATITISTICAL ANALYSIS SYSTEM - SAS. User's} guide. Version 6.4. Cary: 1989. 846 p.

SOUZA, A. D. L. et al. Constituintes químicos de Gustavia augusta L. (LECYTHIDACEAE). Química Nova, v. 24, n. 4, p. 439-442, 2001.

SOUZA FILHO, A. P. S.; DUTRA, S.; SILVA, M. A. M. M. Métodos de superação de dormência de sementes de plantas daninhas de pastagens cultivadas da Amazônia.

Planta Daninha, v. 16, n. 1, p. 3-11, 1998.

TIPPING, P. W. Mowing-induced changes in soil seed banks and populations of plumeless thistle (Carduus acanthoides) and musk thistle (Carduus nutans). Weed Technol., v. 22, n. 1, p. 49-55, 2008.

ZHANG, L. et al. A new sterol from Securidaca inappendiculata. J. Asian Nat. Produc. Res., v. 7, n. 4, p. $649-653,2005$. 